\title{
The Patient Cohort of the German Competence Network for HIV/AIDS (KompNet): A ProfiLe
}

\author{
K. Jansen ${ }^{1}$, C. Michalik ${ }^{2}$, M. Hahn 3 , S. Dupke ${ }^{4}$, S. Esser ${ }^{5}$, H. Jaeger ${ }^{6}$, S. Köppe ${ }^{7}$, A. Moll ${ }^{8}$, A. Plettenberg 9 , \\ R. E. Schmidt ${ }^{10}$, A. Skaletz-Rorowski ${ }^{1}$, N. H. Brockmeyer ${ }^{1,11}$, and the Competence Network for \\ $\mathrm{HIV} / \mathrm{AIDS}$ \\ ${ }^{1}$ Kompetenznetz HIV/AIDS, Ruhruniversität, Bochum, ${ }^{2}$ Zentrum für Klinische Studien, Köln, \\ ${ }^{3}$ Institut für Medizinische Statistik, Informatik und Epidemiologie, Köln, ${ }^{4}$ Gemeinschaftspraxis Driesener Straße, Berlin, \\ ${ }^{5}$ Universitätsklinik, Essen, ${ }^{6} \mathrm{MVZ}$ Karlsplatz, HIV Research and Clinical Centre, München, \\ ${ }^{7}$ Gemeinschaftspraxis Mehringdamm, Berlin, ${ }^{8}$ Praxiszentrum Kaiserdamm, Berlin, ${ }^{9}$ Ifi-Institut, Hamburg, \\ ${ }^{10}$ Medizinische Hochschule, Hannover, ${ }^{11}$ Universitätsklinik, Bochum, Germany
}

\begin{abstract}
Objective: In this paper, we describe the main objectives, the study design and the onset of the patient cohort of the German Competence Network for HIV/AIDS (KompNet). Furthermore, we depict sociodemographic and clinical baseline characteristics and an estimation of the coverage and representativity as to the composition of persons living with HIV/AIDS (PLWHA) in Germany.

Methods: The KompNet cohort is an open, retrospective and prospective, multicenter, disease-specific and nationwide cohort study that started gathering data in June 2004. Semi-annually, follow up visits of the patients are documented, covering clinical and sociodemographic data. At enrolment and three years afterwards, an EDTA-sample is taken; a serum-sample is taken at every follow up visit.

Results: As of 14.9.2008, a total of 15,541 patients were enrolled by 44 documenting sites. In September 2007, the cohort size was reduced to 10 outpatient clinics and fifteen private practitioners, covering a total of 9,410 patients. The documentation of these patients comprises 24,117 years of follow up-time since enrolment (mean: 2.6 years), 62,862 person years inclusive data documented retrospectively on course of HIV-infection and combined antiretroviral therapy (cART, mean: 6.7 years). 1,008 patients $(10.7 \%)$ were lost to follow up and $175(1.9 \%)$ died since enrolment. $84.9 \%$ of patients were men. Main risks of transmission were sex between men (MSM: 62.9\%), heterosexual contacts (18.4\%), intravenous drug use (IVDU: $7.0 \%$ ) and origin from a high prevalence country (HPL: $5.2 \%$ ). Mean age was 45 years.

Conclusion: The KompNet cohort covers about a quarter of all patients being under treatment in Germany. The composition of the cohort represents well the most important risks of transmission in Germany. The cohort contains a high proportion of patients being older than 49 years $(28.1 \%)$. On basis of its comprehensive database and its biomaterials banks, the KompNet cohort serves as an important instrument to monitor and analyse the effects of combined anti-
\end{abstract}

retroviral therapy (cART) in Germany, interdigidating basis, clinical and psychosocial research in view to translational research.

Key words: Cohort Study, HIV/AIDS, Germany, Epidemiology, Clinical Research, Profile, Competence Network HIV/AIDS

\section{INTRODUCTION}

How DID THE STUDY COME ABOUT?

In Germany, the first patients diagnosed with AIDS were reported in 1982; their number rose distinctly within the next years [1]. After introduction of cART, the number of reported AIDS cases and deaths due to AIDS in Germany decreased heavily, in coincidence with an increasing HIV prevalence [2]. At the end of 2008, the number of persons living with HIV in Germany is estimated as 63,500, thereof 10,500 persons with AIDS [3]. The number of patients currently treated in Germany is estimated as 30,000 [4]. In 2008, 2,806 newly diagnosed HIV infections were reported. This number grew constantly between 2001 and 2008 from 1,443 to 2,806 per year, with a flattening since 2006. The epidemiology of HIV/AIDS in Germany is characterised by a high proportion of men. Sex between men (MSM) is the main risk of transmission, reflecting the characteristics of many Western countries. MSM was risk of transmission in $65 \%$ of all reported newly diagnosed HIV-infections in 2008 , followed by heterosexual contacts $(17 \%)$ and persons originating from high prevalence countries $(12 \%)$ [2]. Compared to that, intravenous drug use was of minor importance in Germany $(5 \%)$. New infections were diagnosed predominantly in urban areas. AIDS cases declined from about 2,000 in 1995 to about 550 in 2007, but a high number of unreported cases is presumed [2]. The number of AIDS cases in 2008 is estimated as about 1,100. This high number is supposed to be caused by late testing and a late start of cART. 
cART of HIV/AIDS improved distinctly within the last years, especially in Western countries. Not only many new and potent drugs of well know substance classes as protease inhibitors (PI), nucleoside reverse transcriptase inhibitors (NRTI) and non-nucleoside reverse transcriptase inhibitors (NNRTI) were developed, which built the basis for cART since 1996. Also complete new substance classes were developed and mostly brought into standard medical practice, such as fusion inhibitors, integrase inhibitors, co-receptor antagonists and maturation inhibitors. Therewith, therapy of HIV/AIDS and its effects become complex more than ever.

In Germany, direct costs of cART are covered by health insurances. Medical care for HIV/AIDS in Germany is provided by specialised outpatient clinics as well as private practitioners. A high threshold regarding medical care in Germany are assumed regarding special subpopulations, e.g. due to lacking health insurance of people without legal residence permit or because of cultural barriers $[5,6,7,8]$.

Due to this high complexity of the therapy of HIV/AIDS and their circumstances, there was a big need to establish an instrument to picture the reality of cART and their effects in Germany. The KompNet cohort of HIV-positive patients in Germany provides data with national coverage and an extensiveness of data acquisition that covers all described aspects of interest $[9,10,11,12]$.

In this profile, we bring out the rationale of the KompNet cohort, its methods and organisation and describe selected baseline characteristics of its patients at state of date September 14th 2008.

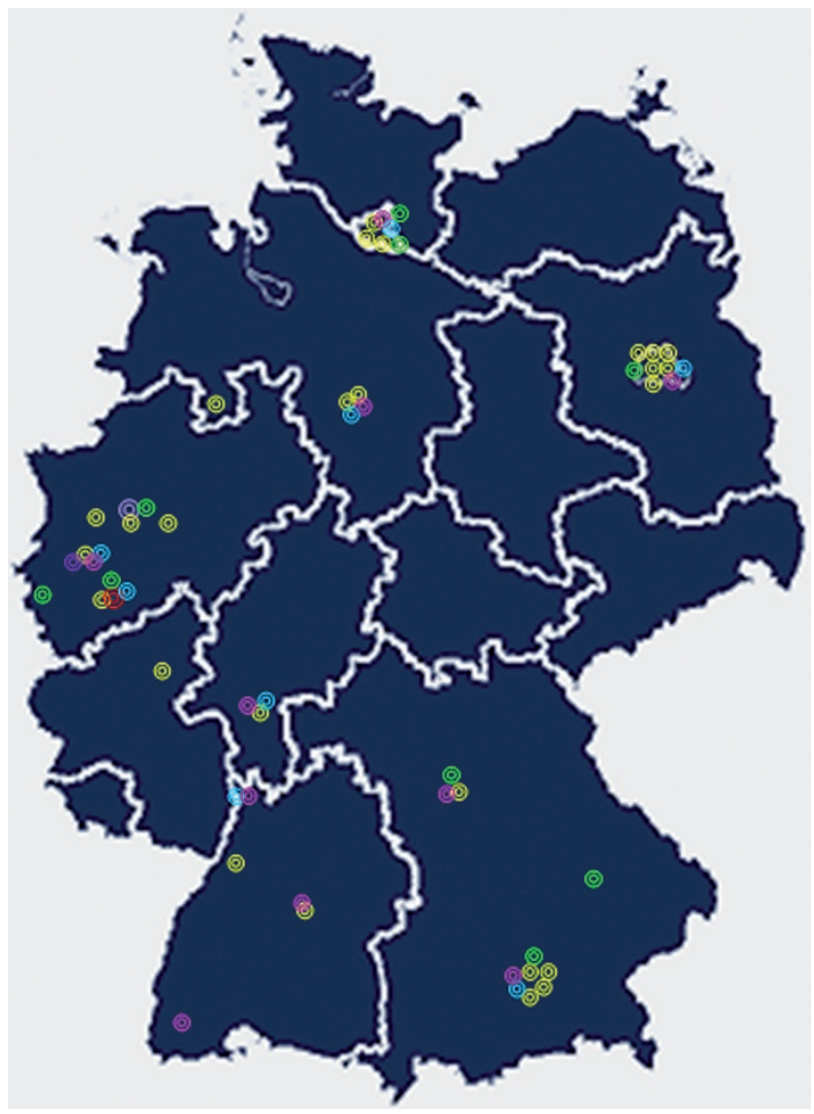

Who SET Up THE KOMPNET COHORT AND HOW IS IT FUNDED?

The KompNet cohort was set up and is conducted by the Competence Network for HIV/AIDS. KompNet was set up in 2002 as a scientific network to foster collaborations in the field of HIV/AIDS research and to bring scientific results into practice. KompNet combines expertise in different disciplines that are represented in four scientific boards: (1) basic research, (2) clinical science, (3) social sciences and public health and (4) gender and pediatric studies. These boards generate scientific projects and evaluate submitted project proposals from outside the network in close collaboration. KompNet is governed by a steering committee. Involving patient interests by including patient representatives into all governmental and scientific bodies of the network and by spreading information actively into the patient community is of high importance within the network.

KompNet is funded by the Federal Ministry of Education and Research from its start in 2002 till August 2010. To ensure its scientific work and to sustain the work with cohort data and biomaterials on a stable basis, KompNet is in transformation into a scientific foundation. KompNet generates its financing out of public funding predominantly and to a lower content out of industrial collaborations, e.g. in the scope of clinical studies.

The infrastructure of the KompNet cohort was established since 2002, enrolment of the first patient was in April 2004.

Basis-Module: 25 sites

- 10 clinics

- 15 special. private practitioners

() Resistance-Module: 9 laboratories

() Children-Cohort: 8 sites

() Pregnancy-Cohort: 10 sites

() Neurology-Module: 1 site

Central Office KompNet

(2) CCT Cologne

Fig. 1. Current composition of documenting sites of the KompNet cohort. 
WHAT ARE THE MAIN OBJECTIVES OF THE KOMPNET COHORT?

The main objectives of the KompNet cohort study are

- to establish a nationwide cohort of HIV-infected patients attending outpatient clinics and private practitioners specialised in treatment of $\mathrm{HIV} / \mathrm{AIDS}$ in Germany, as representatively as possible for the German situation,

- to collect a wide range of clinical and sociodemographic data as well as biomaterials regularly to foster clinical, laboratory and psychosocial research on HIV/AIDS in Germany,

- to monitor the epidemiological composition of patients in German treatment institutions,

- to monitor on national level the course of HIV-infection and the effects of cART, its side effects, concomitant diseases and their treatment and to analyse underlying mechanisms,

- to provide clinical data as well as biomaterials to scientific projects from in- and outside the KompNet and to collaborate with similar projects, on national and international level.

\section{Methods: How ARE DATA MEASURED?}

The KompNet cohort is an open, retrospective and prospective, multicenter, disease-specific and nationwide cohort, starting in 2004. The data come from and are documented by clinical sites all over Germany (outpatient clinics and private practitioners, Fig. 1).

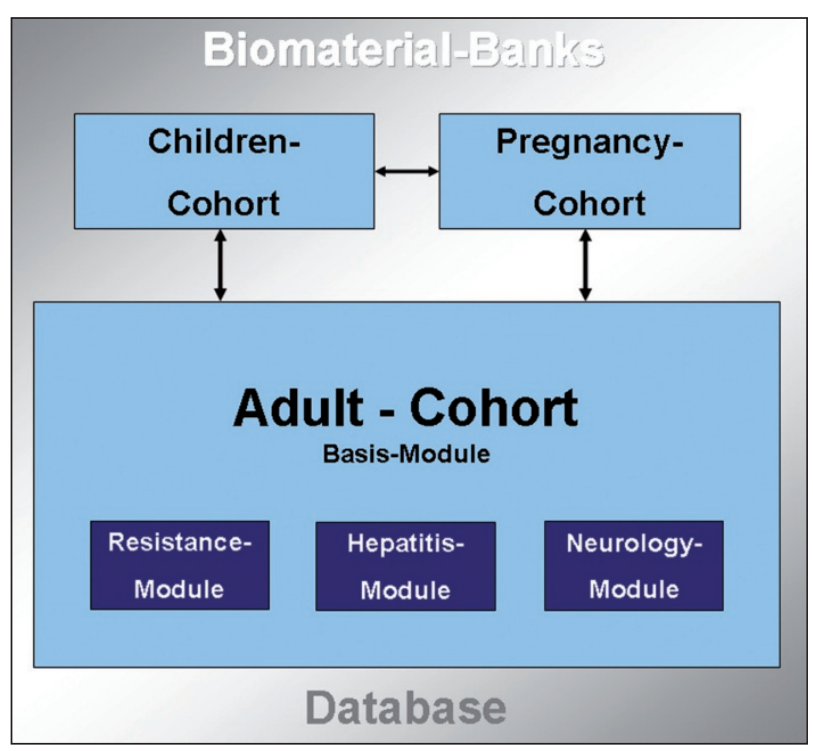

Fig. 2. Modular structure of the KompNet cohort

The cohort consists of several modules, covering different patient populations respectively clinical aspects (Fig. 2). The steering committee decides on the composition of the variable sets applied to document patient data within the different modules. Over time, the variable sets were complemented, especially in regard to enhance sociodemographic and behavioural aspects.
Informed consent forms, specific for the basis module, the children as well as the pregnancy cohort, are used to explain the content of the cohort study, comprehensively and patient-friendly. These forms were approved by the ethic committee of every documenting site. Before participation, each patient has to sign the informed consent.

An extensive data protection concept was evaluated and implemented, on basis of the "generic data protection concept" of the "Telematik-Plattform für medizinische Forschungsnetze in Deutschland" [13]. Every documenting site acts as its own "data trustee", being the only place which holds both, the real name of the patient and his unique patient identifier (PID) of the specific site, used for identification in the scope of the cohort documentation. This is because the treating institution has the most comprehensive protection regarding confiscation of patient data. All other parts of the network dealing with data only use the PID. This concept applies for all modules.

Data are documented via 2 follow up documentations per year. Documentation is done electronically using the GCP- and FDA-approved software MACRO, distributed by InferMed Ltd. Predefined case report forms (CRFs) are used to document data in a highly standardised way. All events occurring meanwhile are documented via a variable number of "cyclic" CRFs. Regarding the basis module, data are documented by documentation officers employed by the documenting sites. In the scope of the children and the pregnancy cohort, data are gained by only one documentation officer travelling to the documenting sites. Data are transferred from the documenting sites encrypted via secured online channels to the central database.

Adult patients are documented within the "basis module". Inclusion criteria is age $>17$ years and a signed informed consent. The documentation includes a comprehensive set of variables, covering clinical and laboratory data and a broad of sociodemographic and behavioural details (s. box). Within the basis module, it is possible to document patient data regarding the time before enrolment into the cohort retrospectively, if such data are available at the documenting site. For this purpose, a core variable set is used covering data on CDC-stage, CD4-cell-count, viral load and data on cART, if the patient was treated.

A serum sample of $4 \mathrm{ml}$ is taken semi-annually at every follow up visit, divided into 4 aliquots and stored decentrally at the documenting site. An EDTA sample of $9 \mathrm{ml}$ is taken at time of enrolment into the cohort and again three years after enrolment, stored centrally after DNA-extraction. Other biomaterials like cerebral spin fluid, lymph node tissue, skin tissue, and mucosa tissue are taken if respective examinations are done, and only consented by the patient. Taking and asservation of biomaterial samples follow detailed standard operation procedures. In case a patient leaves his/her treating institution and change to another that is participating in the KompNet cohort, an ongoing documentation is possible by passing on the specific PID.

Data of HIV-infected and -exposed children are surveyed within the children cohort. Inclusion criteria are age $<18$ years and a signed informed consent. 
There are different informed consent forms for HIVinfected children as well as for parents of HIV-infected and HIV-exposed children. HIV-infected children are followed up till leaving their paediatrician, HIV-exposed children are followed up for 24 month. Data as to the mother and the child (general anamnestic data, HIV anamnesis, data on birth, clinical and laboratory data on HIV and other diagnoses as well as data on cART) are collected semi-annually (for details of documented variables, please see website of KompNet: www.kompetenznetz-hiv.de). At enrolment, a $4 \mathrm{ml}$ serum sample is taken from the child, afterwards $1 \mathrm{ml}$ at each follow up visit. Also at enrolment, an EDTA sample of $5 \mathrm{ml}$ is taken. Actually, eight specialised paediatric HIV-centres participate in the children cohort. Due to the documentation by one travelling documentation officer, it is the aim to cover nearly all HIV-infected and exposed children in Germany over time, also being under medical care not at one of the eight main documenting sites.

HIV-positive pregnant women are followed up for the term of their pregnancy within the pregnancy cohort. Inclusion criteria are a HIV-infection, pregnancy and a signed informed consent. Due to the short time of pregnancy, the data are documented only once. Data on sociodemography, gynaecological anamnesis, the course of pregnancy, the delivery, HIV-anamnesis as well as clinical findings and therapy of HIV and concomitant diseases are collected (for details of documented variables, please see website). At enrolment, a serum sample of $4 \mathrm{ml}$ and a $9 \mathrm{ml}$ sample of EDTA blood are taken.

For both, the children and the pregnancy cohort, a linkage of patient's data to the basis module is possible, so children and pregnant women can be followed up further, if attending one of the documenting sites of the basis module.

Within specific modules, data on hepatitis and neurological impairments of patients included in the basis module can be collected, if existent (for details of documented variables, please see website).

Data on resistant HIV of adult patients are reported by nine laboratories within a resistance module (Fig. 1). Only sequences of clinical routine testing are collected. To link the sequence data to clinical data and to ensure a maximum of data protection, a specific sequence number is used in addition to the PID of the patient to indicate the specific sequence data. Also here, the concept of the treating institution acting as a "data trustee" is applied.

To assure the quality of documented data, several monitoring processes are applied: while electronic data entry, formal implausibilities are checked, documentation of affected data is denied. Via a central monitoring process, more detailed aspects of plausibility of the data are analysed constantly using a programming of predefined criteria that are complemented steadily. In addition, implausible data are identified while conducting scientific analyses. All implausible data including located duplicates are sent to the documenting sites for correction. In case of locating systematic errors in the documentation of a specific site, an on-site monitoring can be conducted. There are trainings of the documentation officers while implementing a doc- umenting site, if a documentation officer changes, and at specific meetings for all documentation officers; additionally, a constant helpline via phone is in use.

\section{RESULTS: HOW IS THE COHORT COMPOSED AND WHO IS IN THE SAMPLE? 1}

\section{Composition}

Enrolment of the first patient took place in April 2004. At start of documentation of data in June 2004, the cohort consisted of 44 documenting sites. A total of 15,541 patients had been registered in the cohort over time. Due to a reduced financing of the cohort, its size had to be scaled down to 26 documenting sites in September 2007. In 2008, another documenting site quit participation. Patients of the excluded documenting sites were no longer followed up since then.

Table 1. Number of enrolled patients, by year and type of documenting site

\begin{tabular}{cccc}
\hline Year & $\begin{array}{c}\text { 25 actually } \\
\text { documenting sites }\end{array}$ & $\begin{array}{c}\text { 19 formerly } \\
\text { documenting sites }\end{array}$ & Total \\
\hline 2004 & 1,991 & 2,161 & 4,152 \\
2005 & 3,966 & 2,851 & 6,817 \\
2006 & 2,531 & 848 & 3,379 \\
2007 & 876 & 271 & 1,147 \\
2008 & 46 & --- & 46 \\
Total & 9,410 & 6,131 & 15,541 \\
\hline
\end{tabular}

Currently, there are 25 documenting clinical sites (Fig. 1), covering 8,227 patients being reported as under follow up ${ }^{2}$. Over time, 1,008 losses to follow up $(10.7 \%)$ and 175 deaths $(1.9 \%)$ were registered. 561 patients (56.7\% of all losses to follow up) were lost to follow up due to a known change to another treating institution not participating in the KompNet cohort. Therewith, the 25 documenting sites cover a maximum number of 9,410 patients. These patients contribute 24,117 person years since enrolment into cohort (mean: 2.6 years) and a total of 62,862 person years including data documented retrospectively (mean: 6.7 years). Due to the reduction of the cohort, a preliminary stop of enrolment was realised in 2008 (Table 1). Since 2009, patients attending a documenting site therapy-naïve can be enrolled retrospectively since January 2008 to ensure coverage of therapy strategies being invented since then.

1 State of date: 14.9.2008. All data on which we refer to further are reported by actually 25 documenting sites of the basis module. Data of excluded sites are handled consistent with the specifications given within the informed consent by the specific patients: in nearly all cases, patients allowed the KompNet to use their data further.

2 In the following, all analyses are made on basis of these 8,227 patients actively under follow up, if not mentioned otherwise. 
The central documentation of HIV-infected and exposed children using the children module started in 2008. Currently, a total of 206 HIV-exposed and 98 HIV-infected children were documented in five of eight documenting sites. Range of age is $0-17$ years. Central documentation of HIV-infected pregnant women will start in 2009 after completion of ethics approval, ten documenting sites are affiliated. Within the neurology module, 92 patients were documented. Documentation within the scope of the resistance module was started in spring 2009, covering 631 sequences actually reported by nine laboratories.

\section{SOCIODEMOGRAPHIC BASELINE CHARACTERISTICS}

With $84.9 \%$, the cohort consists predominantly of men. Men were older than women, regarding the mean age (46 vs. 43 years, $\mathrm{p}<0.001$ ) as well as the mean age at HIV-diagnosis (36 vs. 32 years, $\mathrm{p}<0.001$, Table 2). The cohort comprises a high proportion of patients $\geq 50$ years $(28.1 \%)$ The proportion of $\mathrm{mi}$ - grants (patients with other origin than Germany) was more as double as high in women as in men, with special emphasis on Sub-Saharan countries (Table 3).

The four main risks of transmission within the cohort were MSM (62.9\%), heterosexual contacts $(18.4 \%)$, IVDU $(7.0 \%)$ and origin from a high prevalence country $(5.2 \%)$, with distinct differences between sexes (Table 3). Men had more often a higher vocational degree than women. Regarding their vocational situation, a high proportion of men $(37.0 \%)$ and women $(49.5 \%)$ were not employed.

\section{CLINICAL BASELINE CHARACTERISTICS}

$28.4 \%$ of all patients were documented as positively diagnosed with HIV before 1995. Proportions of men and women regarding the CDC-stage were similar. $12.5 \%$ of all patients had an AIDS event before starting cART.

Of 4,944 patients, the therapy status (therapy-naïve or not) at start of documentation was given. $83.1 \%$ were therapy-naïve at start of documentation, so the complete cART-history of this subpopulation could

Table 2. Actual baseline characteristics of patients of the KompNet cohort actually under follow up, by sex

\begin{tabular}{|c|c|c|c|}
\hline & male & $\begin{array}{l}\text { Sex } \\
\text { female }\end{array}$ & p-value \\
\hline \multicolumn{4}{|l|}{ Number } \\
\hline absolute $^{1}$ & 6,976 & 1,241 & \\
\hline$\%$ & 84.9 & 15.1 & \\
\hline Mean age (years, IQR) & $46(40-52)$ & $43(36-48)$ & $<0.001$ \\
\hline Mean age at HIV-diagnosis (years, IQR) & $36(29-41)$ & $32(25-37)$ & $<0.001$ \\
\hline Mean age at first documented date (years, IQR) ${ }^{2}$ & $39(32-44)$ & $36(29-41)$ & $<0.001$ \\
\hline Mean age at start of initial therapy (years, IQR, $N=3,119$ ) & $40(33-46)$ & $36(29-41)$ & $<0.001$ \\
\hline Highest vocational degree $(\mathrm{N}=6,316)$ & & & $<0.001$ \\
\hline Vocational training & 42.7 & 38.4 & \\
\hline Technical school & 20.9 & 16.1 & \\
\hline University & 26.4 & 13.8 & \\
\hline No vocational degree & 7.7 & 28.1 & \\
\hline Other & 2.3 & 3.6 & \\
\hline Vocational situation $(\mathrm{N}=7,011)$ & & & $<0.001$ \\
\hline Fully employed (> 34 h/week) & 50.7 & 26.6 & \\
\hline Part time work (15-34 h/week) & 6.4 & 16.0 & \\
\hline Employment by the hour ( $<15 \mathrm{~h} /$ week $)$ & 3.5 & 5.8 & \\
\hline Not employed & 37.0 & 49.5 & \\
\hline Other & 2.4 & 2.1 & \\
\hline Mean number of CD4-cell count at start of therapy & $292(159-389)$ & $315(155-423)$ & 0.07 \\
\hline Mean HIV RNA copies at start of therapy $(\log , \mathrm{IQR})^{3}$ & $4.2(3.4-5.2)$ & $3.9(3.0-4.9)$ & 0.001 \\
\hline Mean number of CD4-cell count in actually treated patients (IQR) & $538(348-683)$ & $518(334-669)$ & 0.06 \\
\hline \multicolumn{4}{|l|}{ Proportion of patients having viral load $<$ detection limit } \\
\hline in actually treated patients $(\%)$ & 78.6 & 76.3 & 0.1 \\
\hline $\begin{array}{l}\text { Mean duration between first positive HIV-diagnosis and start of } \\
\text { initial cART (years, IQR) }\end{array}$ & $3.3(0.2-4.7)$ & $3.7(0.2-5.6)$ & 0.06 \\
\hline
\end{tabular}

\footnotetext{
1 Ten patients without data on sex, so $\mathrm{N}=8,217$.

2 Including retrospective data.

3 Viral loads of patients below detection limit were calculated as detection limit-1, depending on the specific test.
} 
Table 3. Comparison of risk of transmission, age, origin and CDC-stage of patients of the KompNet cohort actually under follow up, in comparison to newly diagnosed HIV-infections reported to the Robert Koch-Institute (aggregated 2004-2008), by sex

\begin{tabular}{|c|c|c|c|c|c|}
\hline & \multicolumn{3}{|c|}{ KompNet } & \multicolumn{2}{|c|}{ RKI } \\
\hline & \multicolumn{3}{|c|}{ Sex } & \multicolumn{2}{|c|}{ Sex } \\
\hline & male & female & p-value & male & female \\
\hline \multicolumn{6}{|l|}{ Number } \\
\hline absolute & 6,976 & 1,241 & & 10,501 & 2,359 \\
\hline$\%$ & 84.9 & 15.1 & & 81.7 & 18.3 \\
\hline \multicolumn{6}{|l|}{ Risk of transmission $1,2(\%)$} \\
\hline MSM & 74.1 & --- & $<0.001$ & 65.0 & --- \\
\hline Heterosexual contacts & 11.2 & 58.6 & $<0.001$ & 10.4 & 30.5 \\
\hline High prevalence country & 2.5 & 20.3 & $<0.001$ & 4.8 & 42.4 \\
\hline IVDU & 5.1 & 17.6 & $<0.001$ & 4.9 & 7.7 \\
\hline MTCT & 0 & 0.1 & 0.05 & 0.5 & 2.5 \\
\hline Blood products & 1.8 & 3.3 & $<0.001$ & 0 & 0 \\
\hline Other & 3.9 & 7.4 & $<0.001$ & --- & --- \\
\hline Not known & 7.4 & 10.9 & $<0.001$ & 14.3 & 16.9 \\
\hline Age $(\%)^{2,3}$ & & & $<0.001$ & & \\
\hline$<20$ years & 0.0 & 0.1 & & 3.0 & 8.2 \\
\hline $20-24$ years & 0.7 & 2.3 & & 7.0 & 12.1 \\
\hline $25-29$ years & 3.1 & 5.4 & & 15.6 & 23.3 \\
\hline $30-39$ years & 20.9 & 33.6 & & 36.2 & 32.4 \\
\hline $40-49$ years & 45.8 & 38.9 & & 26.8 & 14.6 \\
\hline $50-59$ years & 19.3 & 13.5 & & 7.5 & 6.2 \\
\hline$\geq 60$ years & 10.2 & 6.2 & & 3.9 & 3.2 \\
\hline Origin $(\%)^{4}$ & & & $<0.001$ & & \\
\hline Germany & 85.6 & 61.8 & & 65.4 & 31.9 \\
\hline Western Europe & 4.2 & 2.8 & & 2.5 & 1.4 \\
\hline Central Europe & 2.4 & 2.4 & & 3.0 & 3.2 \\
\hline Eastern Europe & 1.3 & 3.2 & & 2.1 & 4.4 \\
\hline Sub-Saharan Africa & 2.2 & 18.4 & & 4.5 & 31.8 \\
\hline South/ South-East Asia & 1.2 & 6.7 & & 1.8 & 7.3 \\
\hline Latin America & 1.2 & 1.8 & & 1.4 & 0.8 \\
\hline Other & 1.4 & 2.0 & & 1.7 & 4.2 \\
\hline Not known & 0.5 & 0.9 & & 17.6 & 15.0 \\
\hline CDC-stage $^{3}(\%)$ & & & 0.002 & & \\
\hline A & 28.9 & 33.8 & & 66.5 & 66.6 \\
\hline $\mathrm{B}$ & 45.1 & 42.3 & & 21.4 & 19.6 \\
\hline $\mathrm{C}$ & 26.0 & 23.9 & & 12.1 & 13.7 \\
\hline
\end{tabular}

${ }^{1}$ Multiple answers possible for KompNet cohort.

2 RKI data extracted using SurvStat[14].

${ }^{3}$ RKI data: category " $<20$ " includes also persons of 20 years.

${ }^{4}$ RKI data: reports with given data on CDC-stage $\left(\mathrm{N}_{\text {men }}=6,981, \mathrm{~N}_{\text {women }}=1,529\right)$ [15].

be documented ${ }^{3}$. Of these, $24.1 \%$ (988) stayed therapy-naïve, $75.9 \%$ (abs.: 3,119) started with cART afterwards: $27.4 \%$ remained initially treated, $72.6 \%$ had a follow up-regimen.

3 The therapy status at start of medical care at the actually documenting site is not known for a subgroup of patients, e.g. because the patients were treated in another institution before attending the actually documenting site. Missing data on therapy status at first documentation in the cohort is under ongoing evaluation in collaboration with the documenting sites.
The mean age at initiation of cART differed four years between men and women (Table 2). The duration between the first positive HIV-diagnosis and start of initial cART was 3.4 years, with no significant differences between sexes. Also, we found no significant differences regarding mean CD4-cell count at start of initial therapy between men and women, mean number of HIV-RNA copies/ml at start of initial cART differed slightly (Table 2). Mean number of CD4-cell count in actually treated patients differed not significantly between sexes, as well as the proportions of actually treated patients having a viral load below detection limit (Table 2). 


\section{BIOMATERIAL BANKS}

The KompNet conducts biomaterial banks that are linked to the clinical data of the patient cohort. On that basis, studies using clinical data in combination with biomaterials can be set up, like pharmacogenetic studies $[16,17,18]$. The current numbers of samples are depicted in Table 4.

Table 4. Type of material, number of samples and type of storage of KompNet biomaterial banks

Type of material Number of samples Type of storage

\begin{tabular}{lcc}
\hline Serum & 46,782 & de-centrally \\
DNA & 14,083 & centrally \\
CSF & 1,288 & centrally \\
Skin tissue & 2,500 & centrally \\
Lymphnode tissue & 120 & centrally \\
\hline
\end{tabular}

\section{COVERAGE AND REPRESENTATIVITY}

Due to the specifics of the German health care service, there are outpatient clinics as well as private practitioners specialised in treatment of HIV-positive patients. The coverage of patients by outpatient clinics is assumed as about $30 \%$. Within the KompNet cohort, ten documenting sites are outpatient clinics having 2,146 patients $(26.1 \%)$ under follow up and fifteen with 6,081 patients $(73.9 \%)$, so this reflects well the assumed coverage in Germany. The documenting sites are located all over Germany and represent the epidemiological situation in Germany with high incidences and prevalences in the urban areas of Berlin, Frankfurt, Hamburg, Hannover, Rhine/Ruhr-area, Stuttgart and Munich well (Fig. 1) [2]. Consisting of about 8,000 patients, the KompNet cohort covers approximately a quarter of all treated HIV-positive patients in Germany, that are estimated as about 30,000 in 2008 [4].

There is no reliable data on sociodemographic and clinical characteristics of the total population of patients treated with cART in Germany. As the best approximation to estimate the representativity of the KompNet cohort regarding the German epidemiological situation, we used baseline characteristics of persons being reported as newly diagnosed for HIV to the Robert Koch-Institute (RKI) mandatory between 2004 and 2008, the timeframe in which patients were recruited for the KompNet cohort $[14,15]$.

Data of these two sources can only be compared in a limited way, due to the different survey conditions. The RKI data contain newly diagnosed HIV infections in Germany, reported mandatory by broadly differing kinds of testing institutions, as health care authorities, AIDS help organisations, physicians etc. The KompNet cohort only covers persons that are under medical care. Due to this different recruitment conditions, differences between this populations have to be assumed. This applies especially to the stage of disease. We expect a substantial share of persons being newly diagnosed for HIV and not being in need of immediate treatment. Of these, many could attend institutions of medical care deferred and in a more progressed state of infection. Consequentially, the KompNet cohort covers higher proportions of patients in a more progressed stage of disease in comparison to the respective distribution of the RKI data (Table 3). This difference affects also the age distribution, with more elderly persons in the KompNet cohort.

The proportion of risks of transmission in persons newly diagnosed for HIV varied rather minor over time, MSM remained the highest proportion, by far [2]. Before this background, risks of transmission should be distributed fairly equal in the KompNet cohort, despite the fact that a subpopulation of patients enrolled in the KompNet cohort were diagnosed as HIV-positive before 2004. There is one exception, with special regard to women in the cohort: persons with origin of a high prevalence country. Due to partly lacking permit of residence or differing understanding of disease, there are barriers of access to treating institutions for migrants. Additionally, there are difficulties in recruiting migrants not originating from Western countries for the cohort, being sceptical of participating in such a surveillance system often. Hence, the proportion of migrants and patients coming from high prevalence countries in the KompNet cohort is only half of the proportion of the data on newly diagnosed infections, for both sexes (Table 3). The distribution of countries of origin reflects that as well (Table 3). The KompNet cohort includes nearly no patients who were infected via mother to child transmission (MCTC). One inclusion criteria of the cohort is age $>17$ years, so there are very few eligible patients having this risk of transmission.

Considering the different patterns between the populations of persons being newly diagnosed for HIV and being under medical care, the KompNet cohort represents reasonably the distribution of PLWHA being under medical care in Germany. This applies to central sociodemographic and clinical aspects as well as to the geographical and professional composition of the documenting sites.

\section{WHAT ARE THE MAIN STRENGTHS AND WEAKNESSES?}

The KompNet cohort is the only nationwide operating cohort of HIV-positive patients in Germany collecting also highly detailed data beyond the central aspects of HIV-infection and cART. Also its extensive sociodemographic dataset and the availability of linked biomaterials are valuable features. Estimating the representativity of the KompNet cohort on basis of existent data, the KompNet can be considered as reasonably representative for the German situation, with exception of the subgroup of migrants.

The KompNet cohort contains a high proportion of older patients $(28.1 \% \geq 50$ years $)$. This is an important condition to analyse the effect of cART in an ageing HIV-population. This topic becomes important noticeably, especially in terms of concomitant diseases in the course of HIV-infection, which are represented by the variable set of the KompNet cohort broadly. The collection of data and biomaterials of children 
and pregnant women as subgroups of patients having very special clinical and treatment characteristics is an essential tool to foster translational research and therewith better therapy outcomes for these patients.

Via the PID and by contacting the documenting site, it is possible to identify patients eligible for additional studies, e.g. clinical trials as well as sociodemographic or behavioural studies. Within the informed consent, the patients have the possibility to state their will whether they would like to be contacted for additional studies or not.

A weakness of the KompNet cohort is the laborious documentation of highly detailed data, which means an ongoing high effort in terms of workload and financing to assure the quality of these data. Due to the specifics of the German system of medical care, it is not always possible to follow up a patient who leaves a documenting site and is treated by an institution not participating in the cohort afterwards. This applies especially to the urban areas of Germany with higher numbers of institutions specialised in HIV-treatment and shortens the follow up time of these patients. Another weakness are the lacking documenting sites in Eastern Germany, except Berlin.

Despite these limitations, the KompNet cohort builds an important complement of the German surveillance instruments in the field of HIV/AIDS.

\section{HOW CAN I GET HOLD OF THE DATA AND WHERE?}

The KompNet can provide data and biomaterials of its cohort to all scientists and institutions involved in research in the scope of HIV/AIDS, on national and international level. Scientists apply for data by submitting a short study proposal (please see website for form) that is evaluated and rated as to feasibility, scientific impact and congruency with the aims of the KompNet by its scientific boards, its steering committee and the central office. Potential collaborators are invited to contact the cohort manager (email: klaus.jansen@klinikum-bochum.de, website: kompetenznetz-hiv.de).

\section{Acknowledgments:}

First, we thank all the patients participating in our cohort for providing their data and biomaterials to the cohort. We are grateful for all the work and dedication of the documentation officers and of the heads of our documenting sites. We thank the Ruhr-University Bochum for financial and structural encouragement. Especially, we thank the Federal Ministry of Education and Research for its ongoing financial and ideally support since 2002 .

The following documenting sites contribute data to the basismodule of the KompNet cohort: Gemeinschaftspraxis Driesener Straße, Berlin - Gemeinschaftspraxis Mehringdamm, Berlin - Gemeinschaftspraxis Turmstraße, Berlin - Gemeinschaftspraxis Fuggerstraße, Berlin - Praxiszentrum Kaiserdamm, Berlin - Universitätsklinikum Benjamin Franklin, Charité, Berlin - Universitätsklinik, Bochum - Universitätsklinikum, Dortmund - Universitätsklinikum, Düsseldorf • Universitätsklinik, Erlangen • Universitätsklinik, Essen • HIVCENTER, Universitätsklinikum, Frankfurt • Ifi-Institut, Hamburg - Infektionsmedizinisches Zentrum Hamburg $(\mathrm{ICH})$, Hamburg • Medizinische Hochschule, Hannover •
Praxis Georgstraße, Hannover - Gemeinschaftspraxis Kriegsstraße, Karlsruhe • Städtisches Krankenhaus Kemperhof, Koblenz • Praxis Hohenstaufenring, Köln • Gemeinschaftspraxis Isartorplatz, München - MVZ Karlsplatz, HIV Research and Clinical Centre, München - Praxisgemeinschaft Franz Joseph-Straße, München - Klinikum, Osnabrück • Praxis Schwabstrasse, Stuttgart

\section{LITERATURE}

1. Robert Koch-Institut. HIV und AIDS. Gesundheitsberichterstattung des Bundes. 2006; 31.

2. Robert Koch-Institut. HIV-Infektionen/AIDS: Jahresbericht 2008. Epi Bull. 2009; 21.

3. Robert Koch-Institut. HIV/AIDS in Deutschland - Eckdaten. 2009.

4. Robert Koch-Institut. Changing epidemiology and consequences for prevention. Invited lecture at the SÖDAK 2009, St Gallen.

5. Franz A. Medizinische Versorgung von Menschen ohne Krankenversicherung. In Borde T, David M (Ed.), Gut versorgt? Migrantinnen und Migranten im Gesundheitsund Sozialwesen 2003; 143-152.

6. Kouznetsov L, Kuznetsov AV, Wienecke R, Zippel SA: AIDS awareness among German resettlers from the former Soviet Union: it is time for an immigrant-specific HIV prevention. Eur J Public Health. 2007; 17(4): 403404.

7. Steffan E, Kerschl V, Sokolowski S: Immigration and HIV/AIDS prevention in Germany - an interdisciplinary challenge. Euro Surveill. 2005; 10(1): E050120 4.

8. Robert Koch-Institut. HIV-Infektionen/AIDS-Erkrankungen: Halbjahresbericht I/2007. Epi Bull. 2007; $\mathrm{B} / 2007$.

9. Jansen K, Brockmeyer NH, Haastert B, Baumgarten A, Stoehr A, Behrens G, Jaeger H, Hartl H, Mutz A, SkaletzRorowski A, Esser S, Michalik C, and the Competence Network for HIV/AIDS. Do patients treated by outpatient clinics or by private practitioners face differing treatment characteristics and clinical outcomes in Germany? Eur J Med Res. 2009; Jun 24; 14 (Supl I): 104.

10. Marcus U, Michalik C, Brockmeyer N H, Duglay V, Zeitz M, Lauenroth-Mai E, Hower M, Reuter S, Stellbrink H-J, Rieke A, Ulmer A, Jansen K, and the Competence Network for HIV/AIDS. Late and Early Presenters in the German KompNet Cohort - a comparison of sociodemographic variables. Eur J Med Res. 2009; Jun 24; 14 (Supl I): 17 .

11. Jansen K, Brockmeyer NH, Baudewig M, Walli R, Haastert B, Freiwald M, Harrer T, Staszewski S, Kuhlmann B, Mosthaf F, Schmidt RE, Scholten S, Skaletz-Rorowski A, Esser S, Michalik C, and the Competence Network for HIV/AIDS. Concomitant diseases of HIV-positive persons (PLWHA) in Germany - a comparison between sexes on basis of the KompNet cohort. Eur J Med Res. 2009; Jun 24; 14 (Supl I): 50.

12. Jansen K, Brockmeyer NH, Dlugay V, Stoll M, Volkert R, Goelz J, Buhk T, Carganico A, Kreckel P, Müller M, Skaletz-Rorowski A, Mertens V, Michalik C, and the Competence Network for HIV/AIDS. Clinical outcomes of HIV-positive patients (PLWHA) having different income status: Results of an analysis on basis of the KompNet cohort. Eur J Med Res. 2009; Jun 24; 14 (Supl I): 9.

13. Reng C.-M., Debold P., Specker C. Pommenering K. Generische Lösungen zum Datenschutz für die Forschungsnetze in der Medizin. 2006.

14. Robert Koch-Institut: SurvStat, http://www3.rki.de/SurvStat, State of date: 30.6.2009.

15. Robert Koch-Institut. Oral communication. State of date: 1.7.2009. 
16. Wyen C, Hendra H, Vogel M, Hoffmann C, Knechten H, Brockmeyer NH, Bogner JR, Rockstroh J, Esser S, Jaeger H, Harrer T, Mauss S, van Lunzen J, Skoetz N, Jetter A, Groneuer C, Fätkenheuer G, Khoo SH, Egan D, Back DJ, Owen A; German Competence Network for HIV/AIDS. Impact of CYP2B6 983 $>$ C polymorphism on NNRTI plasma concentrations in HIV-infected patients. J Antimicrob Chemother. 2008. Apr; 61 (4): 914.8.

17. Kreuter A; Wieland U; Gambichler T; Altmeyer P; Pfister $\mathrm{H}$; Tenner-Racz K; Racz P; Potthoff A; Brockmeyer NH. P16ink4a expression decreases during imiquimod treatment of anal intraepithelial neoplasia in HIV-infected men and correlates with the decline of lesional high-risk HPV-DNA load. British Journal of Dermatology. 2007; 157: 523-30.

18. G. Arendt., T. Nolting, C. Frisch, I.W. Husstedt., N. Gregor, E. Koutsilieri, M. Maschke, A. Angerer, M. Obermann, E. Neuen-Jacob, O. Adams, S. Loeffert, P. Riederer, V. ter Meulen, S. Sopper, for the Competence
Network HIV/AIDS, Germany. Intrathecal viral replication and cerebral deficits in different stages of HIV disease. J Neurovirol. 2007; 13(3):225-32.

Address for correspondence:

Dipl.-Psych. Klaus Jansen

Competence Network for HIV/AIDS

Clinic for Dermatology and Allergology

Ruhr University Bochum

Gudrunstr. 56

44791 Bochum

Germany

Tel.: $\quad+49-234-5093501$

Fax: $\quad+49-234-5093475$

Email: klaus.jansen@klinikum-bochum.de

Items of the Basis-Module (variables are described without items regarding dates, units, frequency etc.)

\section{Sociodemographic data}

Vital status, sex, date of birth, place of residence, nationality, ethnicity, country of HIV infection, highest graduation, vocational situation, monthly household netto income, health insurance status, partnership, living situation

\section{Anamnesis}

Family anamnesis: Diabetes mellitus, increased triglyceride, increased cholsterine, cardiovascular diseases, arterial obstructive disease, stroke

HIV anamnesis: date of last negative HIV test, date of first positive HIV diagnosis, HIV subtype, therapy status at enrolment (naïve, treated), assumed date of infection, risk of infection of enrolled patient, risk of infection of infecting partner, infecting partner testes for HIV

\section{Clinical findings}

Height, weight, CDC-classification, weight loss, changes of body shape, clinical diagnoses (AIDS-associated, AIDS defining, concomitant diseases), diarrhoea (stool frequency, medication and side effects)

\section{Laboratory findings}

HIV: CD3-/CD4-/CD8-T-lymphocytes, PCR

Haematology: status at blood withdrawal, haemoglobin, haematocrit, leucocytes, lymphocytes (\%), neutrophiles (\%), thrombocytes, MCV

Clinical chemistry: blood glucose, HbA1c, C-peptide, total cholesterol, HDL cholesterol, LDL cholesterol, VLDL cholesterol, triglycerides, alc. phosphatase, cholinesterase, lactate

Further parameters of infection*: CMV IgG, Toxoplasma gondii IgG, TPHA, VDRL

Metabolic impairments*: rod neutrophiles, segmented neutrophiles, eosinophiles, basophiles, monocytes, lymphozytes, CK, K, Ca, Na, urea, LDH, AST, ALT, $\gamma$-GT, total bilirubin, Lipase, creatinine, albumin, total protein, quick, CRP, bone density, $t$ value, $z$ value

Gastro-enterology*: folate, beta-carotine, vitamine B12, vitamine D3, zinc, test for lactose tolerance, Schilling test, xylose test

Microbiology*: material, pathogen, localisation

\section{Medication}

cART: substances, daily dose, frequency, application, first or proceeded prescription, change, interruption, side effects, reason for change

constant and actual concomitant medications: substance, side effects

\section{Behavioural data}

Consumption of alcohol, amount and frequency (drinks/day), actual and past smoking habit (packyears)

* data on these variables are documented if the regarding examination was done. Otherwise, "not done" is documented. 